\title{
Análise do Comportamento e Cinema: Avaliação das evidências do Efeito Kuleshov e interpretação dos processos comportamentais envolvidos
}

\section{Behavior Analysis and Cinema: Assessment of the evidences supporting the Kuleshov Effect and an interpretation of its behavioral processes}

\author{
Análisis de la Conducta y Cine: Evaluación de las evidencias del Efecto \\ Kuleshov e interpretación de los procesos conductuales implicados
}

Victor Anschau de Biassio ${ }^{1}$, Alexandre Dittrich ${ }^{2}$

Resumo: O Efeito Kuleshov é um processo frequentemente citado e influente no meio cinematográfico. Conforme usualmente descrito, ele consistiria na alteração da percepção de uma situação estimuladora, avaliada por meio de um relato emitido por observadores de uma sequência de fotogramas. O presente artigo tem como objetivo responder a duas perguntas: (1) Quais evidências empíricas sustentam a existência do Efeito Kuleshov? (2) Como este processo pode ser compreendido sob a perspectiva da Análise do Comportamento? O material para análise foi coletado a partir de uma ampla revisão de literatura, contemplando livros especializados em história do cinema, páginas eletrônicas de escolas de cinema renomadas e bases de dados relevantes para a Psicologia. Após a análise do material coletado, concluiu-se que: (1) São escassas as evidências disponíveis sobre os detalhes metodológicos e sobre a própria ocorrência dos experimentos de Lev Kuleshov; (2) Os processos potencialmente envolvidos no Efeito Kuleshov podem ser compreendidos a partir de variáveis respondentes e operantes, destacando a relevância de contingências sócio-verbais e da história de interação individual com as artes cinematográficas. Sugerimos, por fim, a importância de abrir ou ampliar canais de colaboração entre a Análise do Comportamento, a teoria cinematográfica e as artes de modo geral.

Palavras-chave: Efeito Kuleshov; Análise do Comportamento; cinema; interpretação. 


\begin{abstract}
The Kuleshov Effect is an often quoted and influent process in the cinematographic business. As it is usually described, it consists in the alteration of the perception of a stimulating situation, evaluated by a report emitted by observers of a photogram sequence. The present article has the objective of answering two questions: (1) Which empirical evidences support the existence of the Kuleshov Effect?; and (2) How this process can be understood from the perspective of the behavior analysis? The material of analysis was collected from a wide review of literature, contemplating books specialized in cinema history, electronic pages of renowned film schools and databases relevant to Psychology. After the analysis of the collected material, we concluded that: (1) The available evidences about the methodological details and the very occurrence of Kuleshov's experiments are scarce; and (2) The processes potentially involved in the Kuleshov Effect can be understood with the help of respondent and operant variables, emphasizing the relevance of the social-verbal contingences and of the history of individual interaction with the cinematographic arts. We suggest the importance of opening or amplifying the channels of collaboration between Behavior Analysis, the cinematographic theory and the arts in general.
\end{abstract}

Keywords: Kuleshov Effect; Behavior Analysis; cinema; interpretation.

Resumen: El Efecto Kuleshov es un proceso muy citado y influente en el medio cinematográfico. Conforme usualmente descrito, él consistiria en la alteración de la percepción de una situación estimuladora, valorada por medio de un relato emitido por observadores de una secuencia de fotogramas. El presente artigo tiene como objetivo responder a dos preguntas: (1) Cuáles evidencias empiricas que sostenen la existencia del Efecto Kuleshov? y (2) Como este proceso puede ser comprendido por la Análisis de la Conducta? El material para la análisis fue colectado a partir de una amplia revisión de literatura, contemplando libros especializados en historia del cine, páginas electrónicas de escuelas de cine renombradas y bases de datos relevantes para la Psicología. Concluimos que: (1) Son escasas las evidencias disponibles sobre los detalles metodológicos y sobre la propia ocurrencia de los experimentos de Lev Kuleshov; y (2) Los procesos potencialmente envolvidos en el Efecto pueden ser compreendidos a partir de variables respondentes y operantes, destacando la relevancia de contingencias socio-verbales y de la historia de interación individual con las artes cinematográficas. Sugerimos, por fin, la importancia de abrir o ampliar los canales de colaboración entre la Análisis de la Conducta, la teoria cinematográfica y las artes de modo general.

Palabras-clave: Efecto Kuleshov; Análisis de la Conducta; cine; interpretación.. 
Durante a década de 1920, o cinema soviético emergiu com grande popularidade na Rússia (Bruni, 2015). A chamada Revolução Russa era ainda bastante recente (1917), e no ambiente político pós-revolucionário o país foi liderado por Vladimir Lenin. O dirigente soviético era um grande entusiasta do cinema, tendo mesmo afirmado que o cinema seria a mais importante das artes. Durante seu governo, o próprio partido comunista começou a encomendar filmes para os cineastas, de forma que a indústria cinematográfica soviética foi alimentada e incentivada pelo governo (Terry, 2007). Foi em meio a essa crescente atenção devotada ao cinema que, em 1919, o cineasta Lev Kuleshov fundou, em Moscou, o Instituto de Cinematografia Estatal da União Soviética (Hammond, 2011), no qual viria a realizar vários experimentos.

Kuleshov buscava demonstrar que a narrativa cinematográfica era formada principalmente pela justaposição de imagens (Nussinova, 1996). Mais precisamente, ele pretendia mostrar que a montagem $^{1}$ era a ferramenta mais importante do cineasta, de modo que seria preciso intervir nesse processo para produzir os efeitos desejados na audiência. Para Kuleshov, o fotograma ${ }^{2}$ não se resumia a uma foto estática que, quando combinada a outras, reproduzia um movimento. Um fotograma equivaleria a um símbolo, ou a uma letra. Quando o cineasta combinava um fotograma a outro, um sentido era construído, de maneira que a montagem de fotogramas seria equivalente à construção de frases completas, formando a linguagem cinematográfica (Prince \& Hensley, 1992). Além disso, Kuleshov concebia a audiência dos filmes de modo ativo, não passivo. Em sua concepção, a linguagem cinematográfica constituída pelos fotogramas montados pelos cineastas transmitiria um "significado intelectual" para a audiência, que deveria se engajar e participar ativamente para fazer as conexões necessárias para a compreensão dos filmes (Bruni, 2015).

Dado que para Kuleshov o significado percebido em um filme é resultado da montagem de foto-

1 De acordo com Giannetti (2013), montagem é um processo que, por meio da associação de ideias, conecta planos e cenas, gerando ação dramática e compondo a linguagem cinematográfica (p. 136).

2 Cada imagem estática presente em um rolo do filme. gramas, e não dos fotogramas isolados (Prince \& Hensley, 1992), seus experimentos foram planejados para sustentar empiricamente tal tese. Dentre eles, o mais famoso descreveu o que ficou conhecido como Efeito Kuleshov - um processo no qual a audiência teria a percepção de um estímulo alterada devido à justaposição de outro estímulo. Tanto o experimento quanto o processo serão detalhadamente abordados em breve. Contudo, ressaltaremos antes a relevância que o experimento adquiriu para a história da arte.

O Efeito Kuleshov é mencionado e explicado por qualquer livro didático que trate de teoria cinematográfica. Ele é tido como "prova" de que o significado extraído de um filme pela audiência é resultado da ordem dos fotogramas (Prince \& Hensley, 1992). Além disso, é eventualmente citado por cineastas aclamados. Alfred Hitchcock afirmou ter utilizado o Efeito Kuleshov no filme Janela Indiscreta (Bruni, 2015). Sergei Eisenstein também foi influenciado pelos experimentos sobre o Efeito, tendo concluído a partir deles que "o filme ... não é outra coisa senão o método de ordenar as imagens nos sentimentos e na consciência dos espectadores" (Merten, 2010, p. 52). A partir de tal definição, Eisenstein desenvolveu a "montagem de atrações"3 que o tornou famoso (Merten, 2010). A influência do Efeito Kuleshov sobre os cineastas soviéticos é amplamente reconhecida, e Russell (2005) chega mesmo a qualificá-lo como responsável pela origem do cinema soviético. Bruni (2015) menciona os cineastas soviéticos Dziga Vertov e Vsevolod Pudovkin como herdeiros das descobertas teóricas e empíricas de Kuleshov. Vale ressaltar também que o experimento influenciou outros campos além da indústria cinematográfica: ele foi mencionado como influência sobre o trabalho do premiado compositor clássico estadunidense Elliott Carter (Theisen, 2010); sobre a performance de dançarinos (Preston, 2009); e mesmo sobre pesquisas no campo da neurologia (Lee, 2006; Mobbs, Weiskopf, Lau, Featherstone, Dolan, \& Frith, 2006). Assim, pode-se afirmar com segurança que o Efeito

3 Estilo de montagem no qual se "submete o espectador a uma ação sensorial ou psicológica, experimentalmente verificada e matematicamente calculada com o propósito de nele produzir certos choques emocionais" (Saraiva, 2011, p. 120). 
Kuleshov é relevante e influente, gerando impactos consideráveis na arte e na ciência.

A Análise do Comportamento, desde as proposições pioneiras de B. F. Skinner (1938, 1953/2003, 1974), sempre se propôs como uma teoria ampla, que busca compreender todo e qualquer processo comportamental a partir de princípios experimentalmente validados. O Efeito Kuleshov claramente se refere a processos comportamentais, o que permite uma interpretação do mesmo a partir de um ponto de vista analítico-comportamental. Tal interpretação tem também o potencial de abrir vias de colaboração entre a Análise do Comportamento e a teoria cinematográfica - para além das análises funcionais do comportamento de personagens de filmes, que parece constituir um recurso didático bastante frequente, pelo menos no Brasil, uma vez que, por meio dessa estratégia, estudantes aprendem "a aplicar certos conceitos em situações análogas à realidade" (Ribeiro \& de-Farias, 2014, p. iv).

Assim, o presente artigo tem como objetivo responder a duas perguntas: (1) Quais evidências empíricas sustentam a existência do Efeito Kuleshov?; (2) Como este processo pode ser compreendido sob a perspectiva da Análise do Comportamento?

\section{Método}

Para responder às perguntas propostas, inicialmente verificamos a existência de registros na literatura sobre detalhes dos procedimentos empíricos empregados para estudar o Efeito Kuleshov (participantes, método, resultados etc.). A partir de tais informações, buscamos identificar as variáveis comportamentais potencialmente envolvidas na ocorrência do fenômeno, apresentando assim interpretações ${ }^{4}$ fundamentadas na Análise do Comportamento.

As informações pertinentes ao Efeito Kuleshov foram buscadas, inicialmente, em livros especializados em história do cinema, que contivessem re-

4 Utilizamos “interpretação" aqui no sentido originalmente proposto por Skinner (1971, pp. 22-23; 1984, p. 578) e posteriormente adotado amplamente pela comunidade analítico-comportamental (e.g., Donahoe, 2004; Strapasson, Carrara, \& Lopes Jr., 2007): “o uso de termos e princípios científicos para falar de fatos sobre os quais sabe-se muito pouco para tornar possíveis a previsão e o controle" (Skinner, 1984, p. 578). ferências específicas à história do cinema soviético. Os livros selecionados foram: The Oxford History of World Cinema (Nowell-Smith, 1996); Cinema: Entre a Realidade e o Artifício (Merten, 2010); História do Cinema Mundial (Saraiva, 2011); Tudo Sobre Cinema (Hammond, 2011); e Understanding Movies (Giannetti, 2013). Além dos livros, procuraram-se em páginas eletrônicas de escolas de cinema textos que fizessem referência ao Efeito Kuleshov. Tais escolas foram selecionadas tendo como referência duas listas feitas pela revista The Hollywood Reporter (THR). A revista criou uma lista com as 25 melhores escolas de cinema dos Estados Unidos (THR, 2015a) e outra lista com 15 grandes escolas de cinema de outros países (THR, 2015b). Foram analisadas as páginas eletrônicas de todas as 40 escolas de cinema indicadas nessas listas.

Também foi realizada uma revisão de literatura em duas bases de dados relevantes para a Psicologia (PsycINFO e BVS-Psi), a fim de verificar se o Efeito Kuleshov tem sido estudado neste âmbito.

Em todos as fontes mencionadas (livros, páginas eletrônicas, bases de dados) a palavra-chave que orientou as buscas foi "Kuleshov Effect" - ou, quando pertinente, sua equivalente em português (Efeito Kuleshov). Foram selecionados apenas textos que apresentassem uma ou mais das seguintes informações: (1) definição do Efeito Kuleshov; (2) evidências empíricas do Efeito Kuleshov; (3) identificação de variáveis potencialmente relevantes para a interpretação analítico-comportamental do Efeito Kuleshov. ${ }^{5}$

\section{Resultados e Discussão}

\section{Definição do Efeito Kuleshov}

O Efeito Kuleshov foi inicialmente descrito a partir de um experimento no qual um filme era apresentado a uma audiência. Nesse filme, um close do rosto do ator russo Ivan Mozzhukhin, apresentando uma expressão facial considerada neutra, era justaposto a três fotogramas diferentes, contendo, respectivamente, um prato de sopa, um cadáver feminino dentro de um caixão e uma criança brin-

5 Detalhes adicionais sobre os procedimentos e resultados da seleção de textos podem ser obtidos junto aos autores, tendo sido aqui omitidos para tornar o texto mais sintético. 
cando com uma boneca (Giannetti, 2013; Prince \& Hensley, 1992). Assim, o experimento compreenderia a visualização de três cenas pela audiência, com cada uma envolvendo três planos: (1) close - prato de sopa - close; (2) close - cadáver - close; (3) close criança - close. ${ }^{6}$ Em cada uma das cenas, o primeiro e o terceiro planos exibidos são iguais (closes). Contudo, de acordo com relatos históricos sobre o experimento, a audiência teria descrito o terceiro plano como diferente do primeiro, ou atribuído características "emotivas" para o terceiro, exclusivamente devido à influência do segundo plano (sopa, cadáver ou criança) (Theisen, 2010). Em outras palavras, a audiência teria "fornecido um sentido" ao rosto inexpressivo de Mozzhukhin por influência dos estímulos apresentados em conjunto com ele em cada sequência (Bruni, 2015).

A justaposição dessas imagens teria feito com que a audiência apresentasse diferentes interpretações para a expressão neutra do ator entre a primeira e a terceira aparição. Nas três sequências apresentadas, os participantes interpretavam a primeira imagem como se Mozzhukhin estivesse observando algo (Giannetti \& Eyman, 1990, citados por Bruni, 2015). Contudo, quando o close era justaposto a um prato de sopa a audiência relatava que o ator estava com fome diante do segundo close. Quando era justaposto ao cadáver, a audiência relatava que $\mathrm{o}$ ator estava triste. Por fim, quando o rosto era justaposto à imagem da criança brincando, a audiência relatava que $o$ ator estaria expressando uma "alegria paterna" (Prince \& Hensley, 1992). Com isso, Kuleshov afirmou ter comprovado sua tese, de acordo com a qual o significado extraído de um filme não é determinado pelo conteúdo dos elementos isolados presentes na montagem (cenários, atores, ações, etc.), mas pela sua justaposição (Nussinova, 1996).

A importância do experimento residiria no fato de ter demonstrado que a montagem faz com que o público traga à consciência seu próprio conteúdo emocional, e então "projete" esse conteúdo nas rea-

6 As imagens não são reproduzidas aqui porque não encontramos os fotogramas originais. As imagens eventualmente utilizadas para ilustrar o efeito não apresentavam Mozzhukhin, ou exibiam objetos distintos dos descritos acima. O motivo pelo qual diferentes imagens são vinculadas ao Efeito é abordado na sequência do texto. ções do ator, identificando no rosto de Mozzhukhin seus próprios sentimentos. O Efeito Kuleshov provaria que a montagem - definida como a justaposição de imagens - pode provocar reações emocionais na audiência dos filmes (Russell, 2005). Kuleshov afirmava ainda que a emoção provocada na audiência não se originava na performance dos atores, mas sim na justaposição de imagens, pois a audiência construiria um sentido emocional para aquilo que assiste a partir de tal justaposição (Giannetti, 2013).

\section{Evidências Empíricas do Efeito Kuleshov}

A revisão de literatura sugere que, a despeito da fama do Efeito Kuleshov, há poucas informações confiáveis sobre os experimentos que teriam fundamentado sua descrição (Khokhlova, 1996). De acordo com Mobbs et al. (2006), as observações feitas por Kuleshov são de natureza anedótica, e Prince e Hensley (1992) afirmam que o fenômeno supostamente descrito pelo experimento nunca foi cientificamente comprovado. As filmagens dos experimentos se perderam, restando apenas imagens estáticas que mais tarde foram restauradas (Bruni, 2015). Além disso, não há nenhum registro sobre quais foram as condições planejadas por Kuleshov para a realização do experimento (Russell, 2005). A falta de fontes de informação confiáveis tem claro impacto na forma como o experimento é apresentado na literatura especializada, dando margem a versões diferentes a respeito de como teria sido realizado; por exemplo, teriam sido incluídas também imagens de uma porta de prisão e de uma situação amorosa (Saraiva, 2011), do caixão de uma criança (Russell, 2005) e de uma mulher nua deitada em um sofá (Prince \& Hensley, 1992). Há também versões discrepantes com relação ao brinquedo utilizado pela menina: enquanto existem referências afirmando que a garota manipula um urso de pelúcia (Terry, 2007), outras relatam que teria sido uma boneca (Giannetti \& Eyman 1990, citados por Bruni, 2015; Prince \& Hensley, 1992). Prince e Hensley (1992) salientam que não se sabe com precisão quais foram os métodos e procedimentos adotados por Kuleshov. Dessa forma, o experimento nunca foi replicado em sua formulação original, simplesmente porque tal formulação é desconhecida. É possível, portanto, levantar diversos questionamentos relevantes de um ponto de vista experimental: Ele, por 
exemplo, entrevistou os sujeitos individualmente ou em grupo? O que ele lhes disse de antemão a respeito do propósito da apresentação? O que ele contou a eles sobre a natureza da edição ou montagem de filmes, se algo? Qual era a frequência de opiniões outlier, isto é, de pessoas que não acharam que Mozhukin ${ }^{7}$ estava triste por causa da mulher morta? Relatos publicados sugerem que as respostas foram uniformes. Foram mesmo? (Prince \& Hensley, 1992, p. 65) ${ }^{8}$

A literatura sugere, portanto, que são escassas as evidências disponíveis sobre os detalhes metodológicos e sobre a própria ocorrência dos experimentos do cineasta Lev Kuleshov que apoiariam a existência do Efeito que leva seu nome; sugere, além disso, que mesmo que tenham ocorrido, tais experimentos possivelmente apresentaram problemas metodológicos relevantes, demandando a realização de novos estudos para a verificação e compreensão do tal Efeito.

Vale ressaltar que Kuleshov não tinha treinamento experimental como cientista do comportamento, e que vários procedimentos hoje comuns na pesquisa comportamental obviamente não existiam na década de 1920 (Prince \& Hensley, 1992). A despeito da impossibilidade de replicar os detalhes metodológicos do experimento original, tentativas recentes e metodologicamente mais precisas de investigar o Efeito Kuleshov foram realizadas, dentre as quais se destacam as Prince e Hensley (1992) e Bruni (2015). ${ }^{9}$ Os resultados de tais estudos são semelhantes, e sugerem um retrato mais nuançado do fenômeno. A maior parte dos observadores

7 No decorrer da pesquisa, encontramos diferentes grafias para o sobrenome do ator. Utilizamos "Mozzhukhin" no corpo do texto porque foi a grafia mais utilizada nas referências encontradas.

8 Did he, for example, interview the subjects individually or in a group? What did he tell them beforehand about the purpose of the presentation? What, if anything, did he tell them about the nature of film editing or montage? What was the frequency of outlier opinions, e.g., people who did not think Mozhukin was saddened by the dead woman? Published accounts suggest the responses were uniform. Was this so?

9 Apresentamos aqui apenas uma síntese dos resultados dos estudos, omitindo detalhes metodológicos que tornariam o texto demasiadamente extenso. Sugerimos ao leitor eventualmente interessado a consulta direta aos relatos das pesquisas, citados na lista de referências. entrevistados apontou que o rosto do ator apresentava uma expressão neutra, embora alguns tenham identificado emoções com nomes variados. Por exemplo, no estudo de Bruni (2015), em uma sequência que exibia uma faca, a resposta mais comum foi a de que o ator mantinha uma expressão neutra (com uma média de 3,56 em uma escala Likert de 1 a 5); em segundo lugar, os participantes apontavam para "agressão" (média de 1,93), e em terceiro lugar, "tristeza" (média de 1,35).

Algumas conclusões podem ser extraídas a partir das poucas evidências empíricas existentes sobre o chamado Efeito Kuleshov. Em primeiro lugar, parece evidente que a forma como ele é apresentado na literatura especializada em cinema é questionável, na medida em que tende a sugerir que Kuleshov "provou" a existência do efeito, sem que se especifique precisamente como e em que grau ele foi provocado, e mesmo no que ele consiste. Investigações recentes sugerem, além disso, que o vocabulário utilizado para classificar emoções está sujeito a diversas variáveis históricas e contextuais, apresentando considerável variabilidade entre diferentes participantes. Tais conclusões são congruentes com investigações que sugerem que variáveis contextuais são relevantes para a atribuição de emoção a outras pessoas (Carroll \& Russell, 1996; Lee, 2006; Mobbs et al., 2006; Wallbott, 1988).

\section{Possibilidades de Interpretação de Comportamentos Relacionados à Audiência de Cinema Sob a Perspectiva da Análise do Comportamento}

De acordo com Giannetti (2013), as teorias de Pavlov constituíram a fundamentação para os experimentos de Kuleshov. Skinner (1953/2003) apontou a relevância do condicionamento respondente para a compreensão dos efeitos provocados pela arte, ao afirmar que os "efeitos emocionais de música e de pintura são em grande parte condicionados" (p. 62). O mesmo vale para o cinema, na medida em que apresenta formas variadas de estimulação que podem eliciar respostas emocionais eventualmente condicionadas durante a história comportamental dos espectadores.

Nesse tipo de condicionamento, um estímulo neutro adquire a função de eliciar uma resposta de uma mesma classe respondente que um determina- 
do estímulo incondicionado (ou previamente condicionado) inicialmente elicia (Skinner, 1953/2003). No caso do Efeito Kuleshov, a audiência seria exposta a um estímulo supostamente neutro (close do rosto do ator), que seria justaposto a um estímulo incondicionado ou previamente condicionado. Um prato de sopa, por exemplo, pode produzir efeitos incondicionados identificados com palavras como "fome". É pouco provável, porém, que uma única exposição à sequência "close - prato de sopa" confira à segunda exibição do close o poder de eliciar, isoladamente, os respondentes de "fome" - e tal efeito seria facilmente confundido com aqueles provocados pelo próprio prato de sopa, dada a justaposição imediata das imagens. Não obstante, a simples ocorrência de tais respondentes diante do estímulo que usualmente os elicia (prato de sopa) pode por si só aumentar a probabilidade do operante verbal que descreve o sentimento ("fome").

Palavras como "fome", "luto" e "orgulho" são definições abstratas, que podem ficar sob controle parcial de eventos privados. Skinner (1957) aponta que a nomeação de eventos privados é ensinada por uma comunidade verbal previamente treinada. A imagem de um caixão, por exemplo, pode evocar sentimentos relatados como "luto", sendo o caixão um estímulo condicionado, presente em situações nas quais, em certas comunidades, cerimônias funerárias são conduzidas e os sentimentos eliciados são descritos desta forma. O caixão possivelmente não eliciaria sentimentos especialmente conspícuos em integrantes de comunidades que não utilizam caixões em suas cerimônias funerárias, sendo também reduzida, portanto, a probabilidade de operantes verbais descritivos de sentimentos.

A despeito da possibilidade de que respondentes privados influenciem a probabilidade das respostas verbais descritivas de emoções alheias, é importante notar que nos experimentos que buscam descrever o Efeito Kuleshov os participantes são solicitados a descrever os sentimentos que $o$ ator estaria expressando, e não os seus próprios. Portanto, os participantes são instruídos a emitir tatos sob controle de estímulos discriminativos não verbais públicos. Assim, os experimentos sobre o Efeito Kuleshov envolvem interações complexas entre respondentes e operantes. A nomeação de sentimentos supostamente experimentados por um ator parece consistir em respostas operantes verbais (tatos) produzidas por generalização.

Sentimentos e emoções são percebidos e nomeados a partir do treino que uma comunidade verbal realiza com um indivíduo (Skinner, 1957). Tal treino envolve não apenas a nomeação dos sentimentos experimentados pelo próprio sujeito em certos contextos, mas também a nomeação de sentimentos sob controle da observação do comportamento de outras pessoas, incluindo as variáveis antecedentes e consequentes que o afetam. A generalização presente nos experimentos que buscaram reproduzir o Efeito Kuleshov se originaria dessa aprendizagem: ao entrar em contato, por exemplo, com contextos típicos de luto e com o comportamento de pessoas nesse contexto, o indivíduo é ensinado a identificá-los verbalmente ("luto") - e, se convidado a descrever o que sentem as pessoas em tal contexto, pode igualmente emitir tal descrição, a despeito da óbvia impossibilidade de identificar eventuais respondentes privados em outras pessoas. Os efeitos desse aprendizado são então descritos como "Efeito Kuleshov". Ao observar, por exemplo, o close do ator seguido do caixão, o espectador poderia ficar sob controle de semelhanças entre essa justaposição e uma observação efetivamente realizada em sua história, na qual vira um sujeito olhando para um caixão em um contexto denominado como "luto" por sua comunidade verbal (no qual as pessoas "sentiriam luto", estariam "de luto" ou "enlutadas", etc.).

A história de exposição a contingências sócio-verbais de cada indivíduo participante dos experimentos é parte essencial da explicação das características (sejam gerais ou particulares) de sua descrição. Isso inclui não apenas a exposição direta a contingências dispostas por uma comunidade verbal, mas também à própria produção cinematográfica. Tais variáveis históricas podem, portanto, explicar o fato de o Efeito Kuleshov ocorrer em alguns indivíduos e em outros não, assim como o fato de diferentes participantes identificarem diferentes sentimentos (ou mesmo "expressão neutra") quando expostos às sequências de imagens. Relatos sobre os experimentos originais de Kuleshov sugerem, por exemplo, que a justaposição do close com a imagem de uma criança brincando teria sido suficiente para que os participantes identificassem "orgulho paternal". Embora tal descrição obvia- 
mente seja possível, é igualmente possível que outros participantes, expostos a outras contingências, emitissem uma ampla variedade de tatos distintos para "identificar os sentimentos" do ator. Eventuais semelhanças na descrição de sentimentos - por exemplo, participantes identificando "agressão" no rosto justaposto a uma faca, conforme ocorreu no estudo de Bruni (2015) - dependem igualmente de semelhanças nas contingências comumente mantidas em um ambiente sócio-verbal.

Ademais, a história específica de contato de cada participante com filmes em geral supostamente pode influenciar sua descrição. Muitas revoluções ocorreram no cinema desde 1920, de forma que sua linguagem evoluiu de modo considerável. A audiência atual está exposta a uma linguagem cinematográfica distinta da de 1920, e poderia, portanto, responder de maneira bastante diferente às condições experimentais originalmente planejadas por Kuleshov. Como exemplo de desenvolvimento da cinematografia, pode-se citar a revolução que Hitchcock potencializou com o filme Psicose (1960). No filme, existe uma sequência de assassinato que dura cerca de 40 segundos, registrada por 70 posições de câmera. Tantos planos registrados em tão pouco tempo constituíram uma importante inovação, que influenciou cineastas de todo o mundo, e posteriormente fundamentou a produção de videoclipes (Merten, 2010). Os recursos cinematográficos disponíveis na década de 20 certamente limitavam as possibilidades de "expressar emoções" por parte de atores e diretores - e possivelmente determinavam de modo relevante as possibilidades da audiência de identificá-las em peças cinematográficas. Conforme apontamos, a maioria dos participantes nos estudos de Prince e Hensley (1992) e Bruni (2015) apontou que o rosto dos atores apresentava uma expressão neutra, a despeito de ser acompanhado por imagens de objetos que poderiam alterar a probabilidade de respostas descritivas de "contextos" nos quais o ator teria sentimentos eliciados. Presumivelmente, um espectador habituado à linguagem cinematográfica atual teria maior dificuldade de identificar controle contextual sobre os sentimentos do ator em uma simples justaposição de imagens, aumentado a probabilidade de que o fotograma do rosto do ator adquirisse controle sobre a resposta descritiva ("expressão neutra"). Por fim, o grau de exposição à linguagem cinematográfica e às comunidades verbais que ensinam a "interpretá-la" presumivelmente constituem variáveis adicionais relevantes na determinação da identificação de sentimentos no comportamento de atores. Um amante de cinema pode, por exemplo, ser ensinado a identificar as "formas de expressar emoção" típicas do cinema da década de 20 , do cinema produzido em certo país, de uma determinada escola de cinema, etc.

De modo mais amplo, é possível afirmar que o "significado" de um filme é produto arbitrário, estabelecido culturalmente e dependente de uma história de exposição a variadas contingências mantidas por comunidades verbais - certamente envolvendo, em grande medida, relações comportamentais simbólicas. Um símbolo pode ser definido como um estímulo "originalmente neutro que adquire significados arbitrários estabelecidos culturalmente" (Aggio, Almeida, Cortez \& de Rose, 2014, p. 30). A cinematografia, como a arte de modo geral, produz e utiliza-se com frequência de símbolos, alterando assim a probabilidade de respondentes (sentimentos) e de operantes verbais relevantes relacionados a eles.

\section{Considerações Finais}

O presente artigo teve como objetivo (1) identificar quais evidências empíricas sustentam a existência do Efeito Kuleshov e (2) sugerir como este processo pode ser compreendido sob a perspectiva da Análise do Comportamento.

Quanto ao objetivo (1), apontou-se que a forma como o Efeito Kuleshov é apresentado na literatura especializada em cinema é questionável, na medida em que ela tende a sugerir que Kuleshov "provou" a existência do Efeito, sem que se especifique precisamente como e em que grau ele foi provocado, e mesmo no que ele consistiria. São escassas as evidências disponíveis sobre os detalhes metodológicos e sobre a própria ocorrência dos experimentos do cineasta Lev Kuleshov que apoiariam a existência do Efeito que leva seu nome; e mesmo que tenham ocorrido, tais experimentos possivelmente apresentaram problemas metodológicos relevantes. Tentativas recentes e metodologicamente mais precisas de investigar 
o Efeito Kuleshov foram realizadas, e sugerem que o vocabulário utilizado para classificar emoções está sujeito a diversas variáveis históricas e contextuais, apresentando considerável variabilidade entre diferentes participantes. O Efeito Kuleshov, portanto, envolve fenômenos comportamentais complexos, cuja compreensão certamente não é tão simples quanto sugerem os relatos comuns na literatura especializada sobre o referido Efeito.

Quanto ao objetivo (2), foram identificadas, no campo dos comportamentos respondente e operante, variáveis potencialmente relevantes para a compreensão não só dos fenômenos comportamentais abordados por Kuleshov e outros pesquisadores, mas para o comportamento de audiências de cinema em geral. Destacou-se a relevância de contingências sócio-verbais para a compreensão da variabilidade presente nos relatos de sentimentos, estejam eles sob controle de variáveis privadas ou públicas. Relatos de sentimentos necessariamente dependem de uma história de aprendizagem do comportamento de relatar, sob controle de contingências dispostas por uma comunidade verbal específica, com práticas verbais particulares. Além disso, a história específica de contato de um indivíduo com filmes em geral, a evolução das técnicas cinematográficas, o grau de exposição à linguagem cinematográfica e às comunidades verbais que ensinam a "interpretá-la" presumivelmente influenciam de modo relevante a identificação de sentimentos em atores por parte dos indivíduos expostos a filmes.

Dada sua natureza interpretativa, o presente trabalho apresenta evidentes limitações. Parece haver uma notável escassez de dados empíricos de qualidade sobre os fenômenos comportamentais envolvidos no Efeito Kuleshov; portanto, uma identificação mais precisa das variáveis envolvidas demandaria a produção de tais dados. O presente estudo aponta algumas variáveis potencialmente relevantes para eventuais pesquisas dedicadas ao tema.

Embora análises funcionais do comportamento de personagens de filmes pareçam constituir um recurso didático bastante frequente no Brasil (de-Farias \& Ribeiro, 2014, 2016; Ribeiro \& de-Farias, 2014), diálogos específicos entre a cinematografia e a Análise do Comportamento parecem escassos ou inexistentes. Assim, espera-se também que o presente trabalho possa abrir ou ampliar os canais de colaboração entre a teoria cinematográfica e a Análise do Comportamento. Para os analistas do comportamento, o estudo do cinema, enquanto fenômeno cultural de massa, assim como das teorias e técnicas que lhe dão suporte, constitui parte relevante do esforço para a compreensão de fenômenos sociais e culturais. $\mathrm{O}$ mesmo pode ser dito em relação à arte de modo geral - um vasto e relevante campo do comportamento humano, sobre o qual a Análise do Comportamento, não obstante, parece ainda ter um interesse limitado. Esperamos que o presente trabalho possa constituir uma pequena contribuição para a alteração deste quadro.

\section{Referências}

Aggio, N. M., Almeida, J. H., Cortez, M. D., \& de Rose, J. C. (2014). O papel das emoções na aprendizagem do comportamento simbólico. Perspectivas em Análise do Comportamento, 5, 27-39. Retirado de http://media.wix.com/ ugd/89bfd6_e45300ec8fdb4e7e859a6a1f8b87b589.pdf

Bruni, P. T. (2015). Re-examining the Kuleshov Effect. Monografia de graduação, Universidade de Pittsburgh. Retirado de http://d-scholarship. pitt.edu/24986/

Carroll, J. M., \& Russell J. A. (1996). Do facial expressions signal specific emotions? Judging emotion from the face in context. Journal of Personality and Social Psychology, 70, 205-218.

de-Farias, A. K. C. R., \& Ribeiro, M. R. (Eds.). (2014). Skinner vai ao cinema: Vol. 1 (2 $2^{\mathrm{a}}$ ed.). Brasília: Instituto Walden4.

de-Farias, A. K. C. R., \& Ribeiro, M. R. (Eds.). (2016). Skinner vai ao cinema: Vol. 3. Brasília: Instituto Walden 4.

Donahoe, J. W. (2004). Interpretation and experimental-analysis: An underappreciated distinction. European Journal of Behavior Analysis, 5, 83-89. doi: 10.1080/15021149.2004.10446387

Giannetti, L. (2013). Understanding movies. Londres: Pearson.

Hammond, W. (2011). A revolução no cinema russo. In P. Kemp (Ed.), Tudo sobre cinema (pp. 5461). Rio de Janeiro: Sextante. 
Khokhlova, E. (1996). News concerning the 'Kuleshov Effect'. Film History, 8, 365.

Lee, K. H. (2006). Different bold responses to emotional faces and emotional faces augmented by contextual information. Dissertação de mestrado, Universidade de Pittsburgh. Retirado de http://d-scholarship.pitt.edu/9055/

Merten, L. C. (2010). Eisenstein: A revolução de Odessa. In L. C. Merten, Cinema: Entre a realidade e o artifício (pp. 47-56). Porto Alegre: Artes e Ofícios.

Mobbs, D., Weiskopf, N., Hakwan, C. L., Featherstone, E., Dolan, R. J., \& Frith, C. D. (2006). The Kuleshov Effect: The influence of contextual framing on emotional attributions. Social Cognitive and Affective Neuroscience, 1, 95-106. Retirado de https://www.ncbi. nlm.nih.gov/pmc/articles/PMC1810228/ http://www.ncbi.nlm.nih.gov/pmc/articles/ PMC1810228/?tool=pmcentrez

Nussinova, N. (1996). The Soviet Union and the Russian émigrés. In G. Nowell-Smith (Ed.), The Oxford History of World Cinema: Vol. 1 (pp. 162-174). New York: Oxford University Press.

Preston, C. J. (2009). Posing modernism: Delsartism in modern dance and silent film. Theatre Journal, 61, 213-233.

Prince, S., \& Hensley, W. E. (1992). The Kuleshov Effect: Recreating the classic experiment. Cinema Journal, 31, 59-75

Ribeiro, M. R., \& de-Farias, A. K. C. R. (Eds.). (2014). Skinner vai ao cinema: Vol. 2. Brasília: Instituto Walden4.

Russell, M. (2005). The Kuleshov Effect and the death of the auteur. Forum: University of Edinburgh Postgraduate Journal of Culture and the Arts, 1 , 1-17.

Saraiva, L. (2011). Montagem soviética. In F. Mascarello (Ed.), História do cinema mundial (7 ${ }^{\mathrm{a}}$ ed.) (pp. 109-142). São Paulo: Papirus.

Skinner, B. F. (1938). The behavior of organisms: An experimental analysis. New York: AppletonCentury-Crofts.

Skinner, B. F. (1957). Verbal behavior. New York: Appleton-Century-Crofts.

Skinner, B. F. (1971). Beyond freedom and dignity. New York: Alfred A. Knopf.

Skinner, B. F. (1974). About behaviorism. New York:
Alfred A. Knopf.

Skinner, B. F. (1984). Coming to terms with private events. The Behavioral and Brain Sciences, 7, 572-579.

Skinner, B. F. (2003). Ciência e comportamento humano (11 $1^{\mathrm{a}}$ ed.). São Paulo: Martins Fontes. (Original publicado em 1953)

Strapasson, B. A., Carrara, K., \& Lopes Jr., J. (2007). Consequências da interpretação funcional de termos psicológicos. Revista Brasileira de Terapia Comportamental e Cognitiva, 9, 227239. Retirado de https://doi.org/10.31505/rbtcc. v9i2.196

Terry, T. (2007). Kuleshov and Pudovkin introduce montage to filmmaking. In R. F. Gorman (Ed.), Great events from history: The 20th century, 1901-1940: Vol. 4 (pp. 2148-2151). Pasadena, CA: Salem Press.

Theisen, A. (2010). A multifaceted approach to analyzing form in Elliott Carter's Boston Concerto. Tese de doutorado, Florida State University. Retirado de http://fsu.digital.flvc. org/islandora/object/fsu\%3A176104

THR Staff (2015a, Outubro). The top 25 film schools in the United States 2015. The Hollywood Reporter. Retirado de http://www.hollywoodreporter.com/lists/best-film-schools-2015-top-828483/item/ithaca-college-top-25-film-823664

THR Staff (2015b, Julho). Top 15 international film schools revealed. The Hollywood Reporter. Retirado de http://www.hollywoodreporter. com/news/best-film-schools-world-811368

Wallbott, H. G. (1988). In and out of the context: Influences of facial expression and context information on emotion attributions. British Journal of Social Psychology, 27, 357-369.

\section{Informações do Artigo}

\section{Histórico do artigo:}

Submetido em: 23/07/2018

Aceito em: 25/03/2019

Editor associado: Carlos Eduardo Costa 